\title{
Flow field of a diffusion flame attached to a thick-walled injector between two coflowing reactant streams
}

\author{
By F. J. HIGUERA AND A. LIÑ் ÁN \\ ETS Ingenieros Aeronáuticos, Pza. Cardenal Cisneros 3, 28040 Madrid, Spain
}

(Received 2 October 1995 and in revised form 30 July 1996)

The flow field of a diffusion flame attached to a thick-rim injector between two coflowing streams of fuel and oxidiser is analysed in the Burke-Schumann limit of infinitely fast reaction rate. The length of the recirculation region immediately behind the injector and the velocity of the recirculating fluid are proportional to the shear stresses of the reactant streams on the wall of the injector for a range of rim thicknesses, and the structure of the flow in the wake depends then on three main non-dimensional parameters, measuring the gas thermal expansion due to the chemical heat release, the air-to-fuel stoichiometric ratio of the reaction, and the air-to-fuel ratio of wall shear stresses. The recirculation region shortens with increasing heat release, and the position of the flame in this region depends on the other two parameters. An asymptotic analysis is carried out for very exothermic reactions, showing that the region of high temperature around the flame is confined by neatly defined boundaries and the hot fluid moves like a high-velocity jet under a favourable self-induced pressure gradient. The immediate wake is surrounded by a triple-deck region where the interacting flow leads to an adverse pressure gradient and a reduced shear stress upstream of the injector rim for sufficiently exothermic reactions. Separation of the boundary layers on the wall of the injector, however, seems to be postponed to very large values of the gas thermal expansion.

\section{Introduction}

A diffusion flame between a gaseous fuel jet and a stagnant or coflowing gaseous oxidiser is the central element of many combustion devices. The flame is said to be stabilized or attached to the injector if combustion begins immediately upon the reactants coming into contact and the heat transfer toward the injector is important to the process, whereas it is said to be lifted if a region long compared with the size of the jet's cross-section exists between the injector and the base of the flame. The structure of the flow around an attached flame and the limits within which the flame remains attached are obviously of great practical importance and have been very much studied. Thus the region at the base of an attached flame of methane or hydrogen in air has been experimentally investigated by Robson \& Wilson (1969), Kawamura \& Asato (1975), Kawamura, Asato \& Mazaki (1980), and Takahashi et al. (1984), among others, for a variety of fuel jet shapes and exhaust conditions, including cases with a coflowing air stream around a thin-walled injector. The velocity, temperature and reactants concentration fields were measured, as well as the position of the flame, the heat release, and the heat fluxes from the flame to the injector and 
to the gas downstream. Models of the lifting mechanism have been proposed based on the idea put forward by Gaydon \& Wolfhard (1953) that, even for an attached flame, molecular mixing of the reactants between the injector and the base of the flame leads to a small volume of combustible mixture where a premixed flame front can propagate toward the injector against the flow. A steady attached flame will exist if the propagation velocity of this front can balance the velocity of the flow. Then a fraction of the heat released at the front is transported downstream, serving as an ignition source for the rest of the flame. If such a balance is not possible in the vicinity of the injector rim, the flame will either lift to an equilibrium position far above the injector or disappear altogether. Importantly, while the flow at the base of a lifted flame is typically turbulent, that in the attachment region is always laminar, even if the jet becomes turbulent further downstream.

The immediate wake of a thick injector rim between two coflowing reactant streams provides the moderate strain rates necessary for a diffusion flame to exist, and also, in the vicinity of the rear stagnation point, the low velocities necessary for a premixed flame to propagate without being blown out by the flow. In addition, depending on the conditions of the reactant streams and the position of the flame, the reverse flow in the recirculation region may also provide an alternative mechanism of flame stabilization that does not depend on the propagation of a premixed flame front. The effect of the injector port thickness was first investigated by Vranos, Taback \& Shipman (1968) for the flame between coflowing jets of hydrogen and air, who found that the range of fuel and air velocities over which the flame remains attached is very much enlarged when that thickness is increased. They reported a variety of flame behaviours, including a second type of lift-off in which the flame is locally extinguished at some distance downstream of the injector leaving a residual attached flame, and related the existence of these flames, and of the enlarged stability domain, to the recirculating eddies behind the injector rim and to the high diffusivity of the hydrogen. The same trends were observed by Yoon, Donbar \& Driscoll (1994) in more recent experiments on supersonic coflowing jets of hydrogen and air. These authors found that the propagating front at the beginning of their attached flames sits in the outer part of the shear layer between the air and the recirculating fluid, where the flow velocity is larger than the propagation velocity of the corresponding stoichiometric premixed flame, and attributed the existence of a steady front under these conditions to a local excess of enthalpy in the recirculating flow, due to the high diffusion flux of hydrogen toward the partially burnt recirculating fluid. In their experiments with methane-air flames, Takahashi \& Schmoll (1990) observed a third type of flame lift-off, characteristic of thick injector rims, whereby the upstream end of the flame becomes oscillatory immediately before lifting. They showed experimentally that the oscillations are associated with the end of the flame moving in and out of a recirculation eddy in the wake of the injector rim.

In this paper numerical and asymptotic techniques are used to analyse some of these features in their simplest form. For this purpose we consider an injector of thickness large compared with the quenching distance of the flame, which is the size of the region round the tip of a thin-walled injector affected by heat conduction from an attached flame, but still sufficiently small for the lateral displacements of the reactant streams to be negligible. In this range of thicknesses the oncoming streams are characterized only by the values of the shear stresses on the injector wall immediately upstream of its end, and both the length of the recirculation region and the velocity of the recirculating fluid scale with these values. In addition, since the emphasis is on the description of the flow field, the chemistry is simplified by 
assuming that the flame is an infinitely thin reaction sheet. This corresponds to the Burke-Schumann limit of infinitely fast reactions in which only two parameters are left to characterize the combustion, namely the air-to-fuel mass stoichiometric ratio and the ratio of heat release to thermal enthalpy. The numerical and asymptotic results presented below show that the second of these parameters affects very significantly the flow, making the recirculation region shorter than for the corresponding isothermal flow and eventually leading to a region of favourable pressure gradients downstream of the recirculation bubble, where a jet-like velocity profile develops in the hot fluid around the flame. The position of the flame in the wake depends mainly on the stoichiometric ratio (that, by this means, also affects the flow) and on the ratio of air to fuel shear stresses. The first of these parameters is determined by the nature of the reactants and their concentrations in the oncoming streams, whereas the second depends on the configuration of the injector and the flow rates of the reactants. Though the assumption of infinitely fast reaction is not appropriate to determine the stability limits, because it always leads to an attached flame, a number of qualitative conclusions on the likelihood of the different types of flame lift-off are drawn by examining the distributions of velocity and strain rate on the flame and their dependence on the parameters mentioned before.

\section{Formulation}

The flow in the attachment region of the flame is nearly planar, independently of the shape of the injector, owing to the small size of this region compared to the size of the injector cross-section. As a model of this flow we consider two parallel streams of density $\rho_{\infty}$, temperature $T_{\infty}$, and velocities $U_{F}$ and $U_{o}$ much smaller than the sound speed in either fluid, carrying mass fractions $Y_{F_{\infty}}$ and $Y_{o \infty}$ of fuel and oxygen, respectively, and separated by a wall of thickness $2 h$. The two streams can either extend to infinity or be confined to finite-width channels formed by the separating wall and two other plane parallel walls, or an outer wall and a symmetry plane. The two streams come into contact at the end of the separating wall, reacting in a flame that releases an energy $Q$ per unit mass of fuel consumed. The chemical reaction is supposed to be infinitely fast, so the flame is a continuous, infinitely thin sheet separating the two reactants, which only coexist in the interior of the flame with infinitely small concentrations (see, e.g., Williams 1985).

Counterflow heat conduction toward the separating wall is important in small regions of size $R e^{-1 / 2} \delta$ about its edges, where the full Navier-Stokes equations should be solved to describe the flow. Here, using for definiteness the conditions of the fuel stream, $\delta$ is the thickness of the fuel boundary layer at the end of the separating wall, or the width of the fuel channel in the case of a developed, parabolic flow (in which case $U_{F}$ and $U_{o}$ are the mean velocities of the streams), $R e=\rho_{\infty} U_{F} \delta / \mu_{\infty}$ is the Reynolds number, which will be assumed to be large, and $\mu_{\infty}$ is the viscosity coefficient at temperature $T_{\infty}$. In what follows a $h \gg R e^{-1 / 2} \delta$ is assumed. Then the balance of convection, with a velocity of order $U_{F} h / \delta \ll U_{F}$ when the separating wall is much thinner than the boundary layers or the channel width, and cross-stream diffusion over a thickness of order $h$ determines the characteristic length $l=\operatorname{Re} \delta(h / \delta)^{3}$ of the near-wake region directly affected by the finite thickness of the wall, which will be the main region of concern here. Since $l \gg h$ for $h \gg R e^{-1 / 2} \delta$, the boundary layer approximation can be used to describe the flow in this region. 
Assuming that the Lewis numbers of the fuel and the oxygen are equal to unity, the non-dimensional energy and species conservation equations are

$$
L(T)=q w, \quad L\left(Y_{F}\right)=-w, \quad L\left(Y_{o}\right)=-S w,
$$

where $w\left(Y_{F}, Y_{o}, T\right)$ is the reaction rate scaled with $h^{2} / \mu_{\infty} Y_{F \infty}, q=Q Y_{F \infty} / c_{p} T_{\infty}$ is the non-dimensional heat of reaction, $S$, the stoichiometric ratio, is the mass of oxygen mixture needed to burn unit mass of fuel mixture, and $L \equiv \rho v \cdot \nabla-(1 / \operatorname{Pr}) \partial / \partial y(\mu \partial / \partial y)$, with $v=(u, v)$ and $\nabla=(\partial / \partial x, \partial / \partial y)$, is the transport operator in the boundary layer approximation. Here $x$ is the streamwise distance from the end of the wall, $y$ is the transverse distance from the centre of the wall toward the oxygen side, $u$ and $v$ are the corresponding velocity components, and $\mathrm{Pr}$ is the Prandtl number, assumed to be constant. The variables $\left(x, y, u, v, p, \rho, T, Y_{F}, Y_{o}, \mu\right)$, where $p$ is the pressure variation from the ambient or background value $R_{g} \rho_{\infty} T_{\infty}$, are scaled with their characteristic values

$$
\frac{\rho_{\infty} \lambda_{F 0} h^{3}}{\mu_{\infty}}, \quad h, \quad \lambda_{F 0} h, \quad \frac{\mu_{\infty}}{\rho_{\infty} h}, \quad \rho_{\infty} \lambda_{F 0}^{2} h^{2}, \quad \rho_{\infty}, \quad T_{\infty}, \quad Y_{F \infty}, \quad Y_{\infty \infty}, \quad \mu_{F},
$$

respectively, where $\lambda_{F 0}=-(\partial u / \partial y)\left(x=0^{-}, y=-h\right)$, i.e. in the fuel stream immediately ahead of the trailing edge.

As can be seen by linearly combining equations (2.1), the mixture fraction and the excess of enthalpy,

$$
Z=\frac{S Y_{F}-Y_{o}+1}{1+S} \text { and } H=T-1+\gamma\left(Y_{F}+Y_{o}-1\right), \text { with } \gamma=\frac{q}{1+S},
$$

are transported as passive scalars $[L(Z)=L(H)=0]$, and they take the values $Z=H=0$ in the oxygen stream and $Z=1, H=0$ in the fuel stream. With these boundary conditions $H=0$ everywhere in the boundary layer approximation.

Adding the supplementary relation

$$
Y_{F} Y_{o}=0,
$$

which is the limiting form of each of equations (2.1) for infinitely fast reaction rate (i.e. $w=0$ to leading order; the two reactants do not coexist), determines the temperature and reactant concentrations in terms of the mixture fraction:

$$
\left.\begin{array}{c}
Y_{F}=0, \quad Y_{o}=1-\frac{Z}{Z_{s}}, \quad T=1+\gamma \frac{Z}{Z_{s}} \quad \text { for } \quad 0 \leqslant Z \leqslant Z_{s}, \\
Y_{F}=\frac{Z-Z_{s}}{1-Z_{s}}, \quad Y_{o}=0, \quad T=1+\gamma \frac{1-Z}{1-Z_{s}} \quad \text { for } \quad Z_{s} \leqslant Z \leqslant 1,
\end{array}\right\}
$$

where $Z_{s}=1 /(1+S)$ and the flame sheet (where $Y_{F}=Y_{o}=0$ ) coincides with the unknown surface $Z=Z_{s}$. On this surface the temperature takes its maximum value, equal to the adiabatic flame temperature $T_{e}=1+\gamma$.

For sufficiently small values of $h$, the problem in the wake region, which is sketched in figure 1 , reduces to determining the distributions of $Z$, the velocity and the pressure using (2.5) and the equations

$$
\begin{aligned}
& \nabla \cdot(\rho v)=0, \\
& \rho v \cdot \nabla u=-\frac{\mathrm{d} p}{\mathrm{~d} x}+\frac{\partial}{\partial y}\left(\mu \frac{\partial u}{\partial y}\right),
\end{aligned}
$$



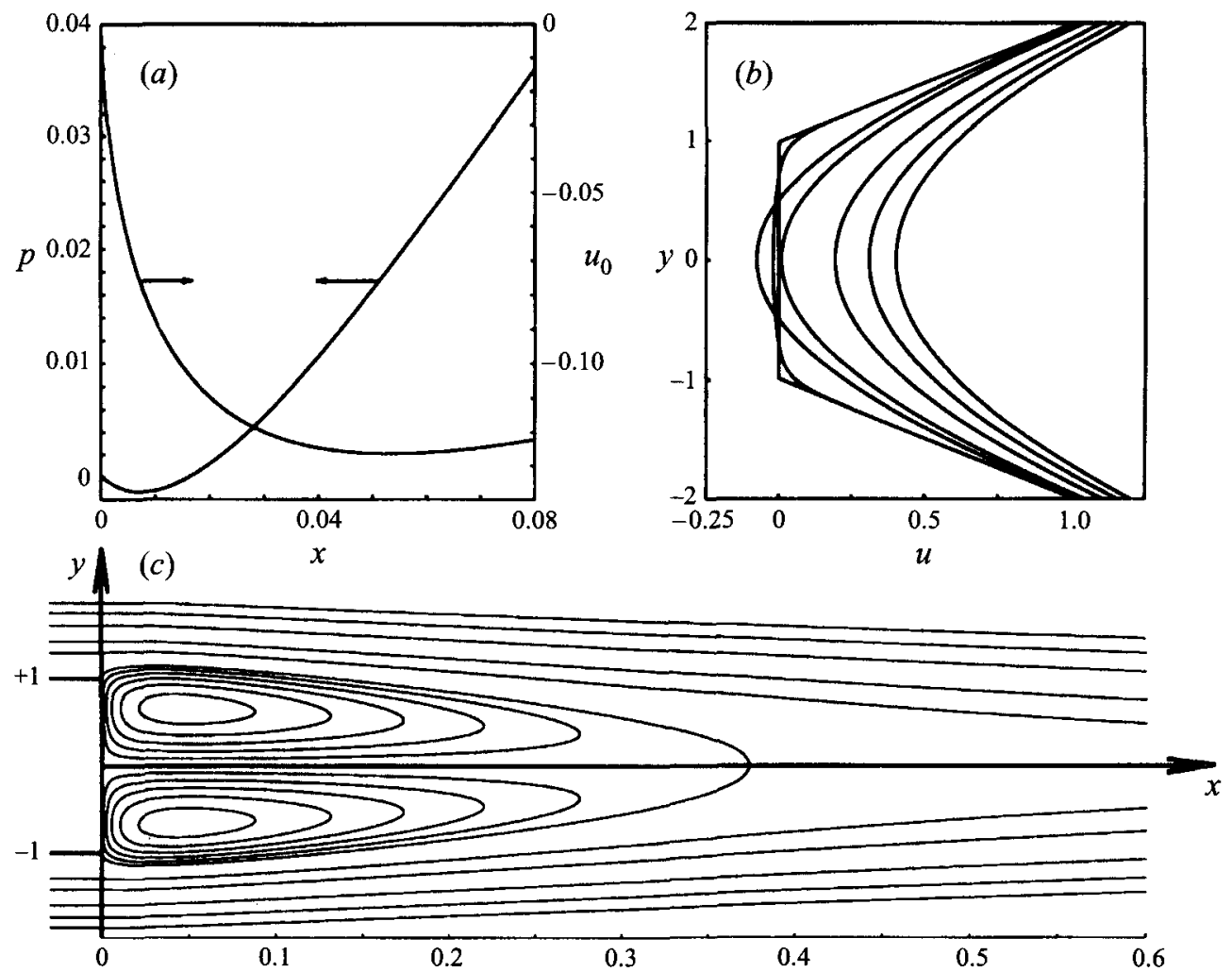

FIGURE 1. Definition sketch. (a) Pressure and velocity along the $x$-axis in the vicinity of the solid. (b) Velocity profiles at $x=0$ (piecewise linear), $6.5 \times 10^{-4}, 0.2,0.4,1.5$, and 2. (c) Streamlines for a constant-density fluid. Plotted are $\psi=0, \pm 0.01, \pm 0.02, \pm 0.03, \pm 0.04$ (in the recirculation region only), and $\pm 0.05, \pm 0.1, \pm 0.2, \pm 0.3$ and \pm 0.4 .

$$
\begin{aligned}
& \rho v \cdot \nabla Z=\frac{1}{\operatorname{Pr}} \frac{\partial}{\partial y}\left(\mu \frac{\partial Z}{\partial y}\right), \\
& \rho T=1, \quad \mu=T^{\sigma} \text {, } \\
& x=0:\left\{\begin{array}{lll}
u=\alpha(y-1), & Z=0 & \text { for } y>1 \\
u=0 & & \text { for }-1<y<1 \\
u=-(y+1), & Z=1 & \text { for } y<-1,
\end{array}\right. \\
& \left.\begin{array}{lll}
y \rightarrow \infty: & u=\alpha(y-1)+o(1), & Z=0, \\
y \rightarrow-\infty: & u=-(y+1)+o(1), & Z=1 .
\end{array}\right\}
\end{aligned}
$$

Here, the boundary conditions at $x=0$ and for $y \rightarrow \pm \infty$ state the matching of the wake with the bases of the oncoming streams ahead and on the sides. The parameter $\alpha$ is the ratio of the shears at the bases of the air and fuel streams. In the equation of state pressure changes have been neglected, which amounts to leaving out acoustics, as well as differences in molecular masses of the reactants (which might not be acceptable for mixtures of hydrogen, for which the assumption of a Lewis number equal to the unity is also inappropriate). The viscosity has been supposed to be the same for the two fluids and to depend potentially on the temperature, with $0 \leqslant \sigma \leqslant 1$ in (2.9).

The conditions (2.11) of zero displacement of the velocity profiles above and below the wake determine the pressure distribution and the overall lateral shift of the wake. 
These conditions hold for sufficiently small values of $h$. Thus, for unconfined streams, a displacement of order $h$ would lead to a velocity perturbation of order $U_{F} h / l$ in the outer flow, and to a pressure perturbation of order $\rho_{\infty} U_{F}^{2} h / l$, which is much larger than the dynamic pressure of the flow in the wake, of order $\rho_{\infty}\left(U_{F} h / \delta\right)^{2}$, if $h \ll R e^{-1 / 4} \delta$. Similarly (2.11) hold for a confined stream when $h \ll R e^{-2 / 7} \delta$; see Smith (1982) for details.

In addition, however small $h$ may be, the release of the no-slip condition at the end of the separating wall and the gas thermal expansion due to the combustion lead to a viscous inviscid interaction region around the end of the wall. In the case of unconfined streams this region is a triple deck of length $\operatorname{Re}^{1 / 4} \delta$ where the pressure perturbations generated in the uniform streams by the evolution of the viscous layer are sufficiently large to affect the flow in a lower deck of thickness $R e^{-1 / 4} \delta$ both upstream and downstream of the end of the wall (Stewartson 1969; Messiter 1970). In the case of confined, developed streams, the length of the interaction region is $\operatorname{Re}^{1 / 7} \delta$, the thickness of its lower deck is $\operatorname{Re}^{-2 / 7} \delta$, and the pressure variations are due to the curvature of the streamlines in the bulk of the channel (Smith 1982). The assumption above, that $h \ll R e^{-1 / 4} \delta$ for unconfined streams or $h \ll R e^{-2 / 7} \delta$ for confined streams, implies that the near wake is much smaller than the lower deck of the interaction region and is embedded in it. Then, in the absence of boundary layer separation, which apparently does not occur in many situations of practical interest, the effect of the interaction region on the near wake is to provide an overall scale factor, determined by the precise value of $\lambda_{F 0}$, which is different from its value at a distance of order $R e^{1 / 4} \delta$ or $R e^{1 / 7} \delta$ upstream.

In what follows the problem (2.5)-(2.11) will be numerically solved for different values of the parameters $\gamma, Z_{s}, \alpha, \sigma$ and $\operatorname{Pr}$ by means of a finite difference method that allows for regions of reverse flow, and the asymptotic limit $\gamma \gg 1$ of very exothermic reactions will be analysed.

\section{Results}

Figures 2 and 3 show some streamlines and isotherms of the flow for $\sigma=0$, $\operatorname{Pr}=1$, and two different values of the exothermicity $\gamma$, the stoichiometric ratio $S$, and the ratio of oxygen-to-fuel shear $\alpha$, which are representative of the range of results obtained. The thick curves give the position of the flame. The wall extends from -1 to +1 on the vertical axis. A region of reverse flow appears behind the wall in all the cases, which for $\alpha=1$ (cases $a$ to $d$ ) is approximately centred (the flow for $\alpha=S=1$ is perfectly symmetric; see figure 1 for an example) and, except in case (d) $(S=8, \gamma=6)$, has two contrarure-rotating eddies. For $\alpha=0.2$ (cases $e$ to $h)$ the region of reverse flow is shorter and less symmetric, having only a smaller clockwise rotating eddy on the side of the slow stream. The length of the region of reverse flow decreases with increasing $\gamma$ (notice the tenfold magnification of the horizontal scales of the figures with $\gamma=6$ relative to those with $\gamma=1$ ) and, for $\alpha=1$, decreases faster for larger values of $S$, which seems to be an effect of the additional asymmetry induced by the lateral displacement of the flame. This length decreases even faster when the increase of the transport coefficients with temperature is taken into account $(\sigma>0$ in (2.9)). The distance from the wall to the farthest point of reverse flow is given in figure 7 below as a function of $\gamma$ for different values of $S$ and $\sigma$. The isotherms in figure 3 are nearly parallel everywhere except in the immediate vicinity of the wall and, when $S$ is high (cases $c, d, g$ and $h$ ), the temperature decays faster toward the upper (air) stream than toward the lower (fuel) stream. 

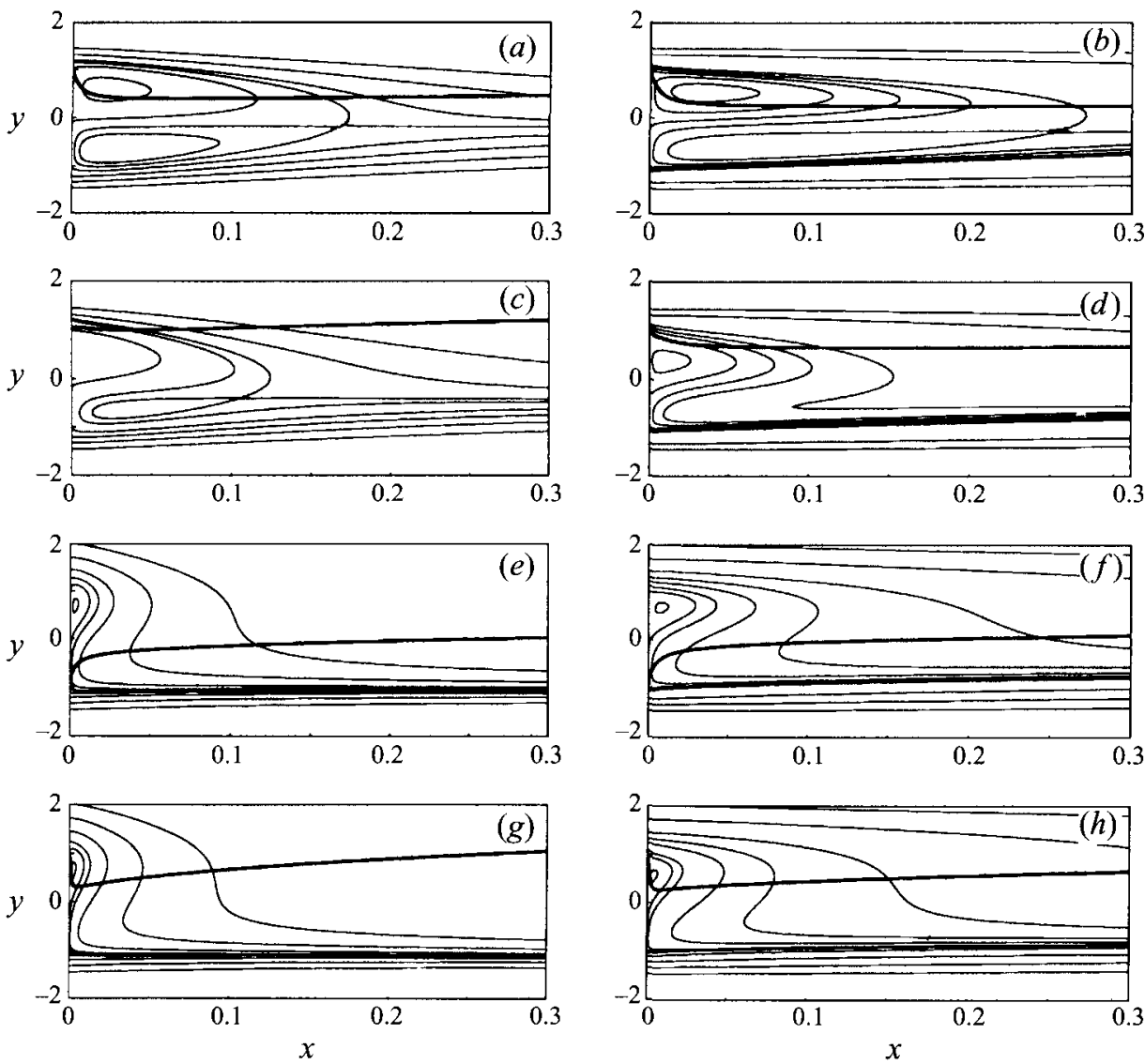

Figure 2. Streamlines and flame for $\sigma=0, \operatorname{Pr}=1$. Values of $(\alpha, S, \gamma)$ are: $(a)(1,2,1)$; (b) $(1,2,6) ;(c)(1,8,1) ;(d)(1,8,6) ;(e)(0.2,2,1) ;(f)(0.2,2,6) ;(g)(0.2,8,1) ;(h)(0.2,8,6)$.

The flame always starts at one of the edges of the wall in the present approximation, except in the strictly symmetric case mentioned before, and proceeds to the lowvelocity wake unless $S$ is very large. For $\alpha=1$ the flame always enters one of the eddies, to an extent that depends on $S$. Thus it reaches the region of negative velocities (toward the wall) for $S=2$ (cases $a$ and $b$ ), whereas for $S=8$ (cases $c$ and $d$ ) more oxygen is needed and the flame shifts toward the air stream, sitting in the upper part of the eddy where the velocity is positive. This situation changes for $\alpha=0.2$. Then the flame with $S=2$ (cases $e$ and $f$ ) starts at the lower (fuel) edge of the wall and never enters the recirculating eddy, having only a short region of negative velocities, whereas with $S=8$ (cases $g$ and $h$ ) it crosses the eddy through the region of negative velocities. The importance of these differences in connection with the possible extinction of the flame will be discussed in $\$ 5$.

The effect of the buoyancy in the region of concern is measured by the Froude number $F r=\mu_{\infty} \lambda_{F 0} / \rho_{\infty} g h$, if the two streams move vertically and have the same density. This effect may be important when the reaction is sufficiently exothermic. Numerical solutions of the problem with a term $(1-\rho) / F r$ added to the right-hand side of (2.7) (corresponding to upward moving streams) show that the buoyancy shortens the recirculation region, specially on the side of the flow containing the flame, which is the more sensitive. Among other things, this selective shortening may 

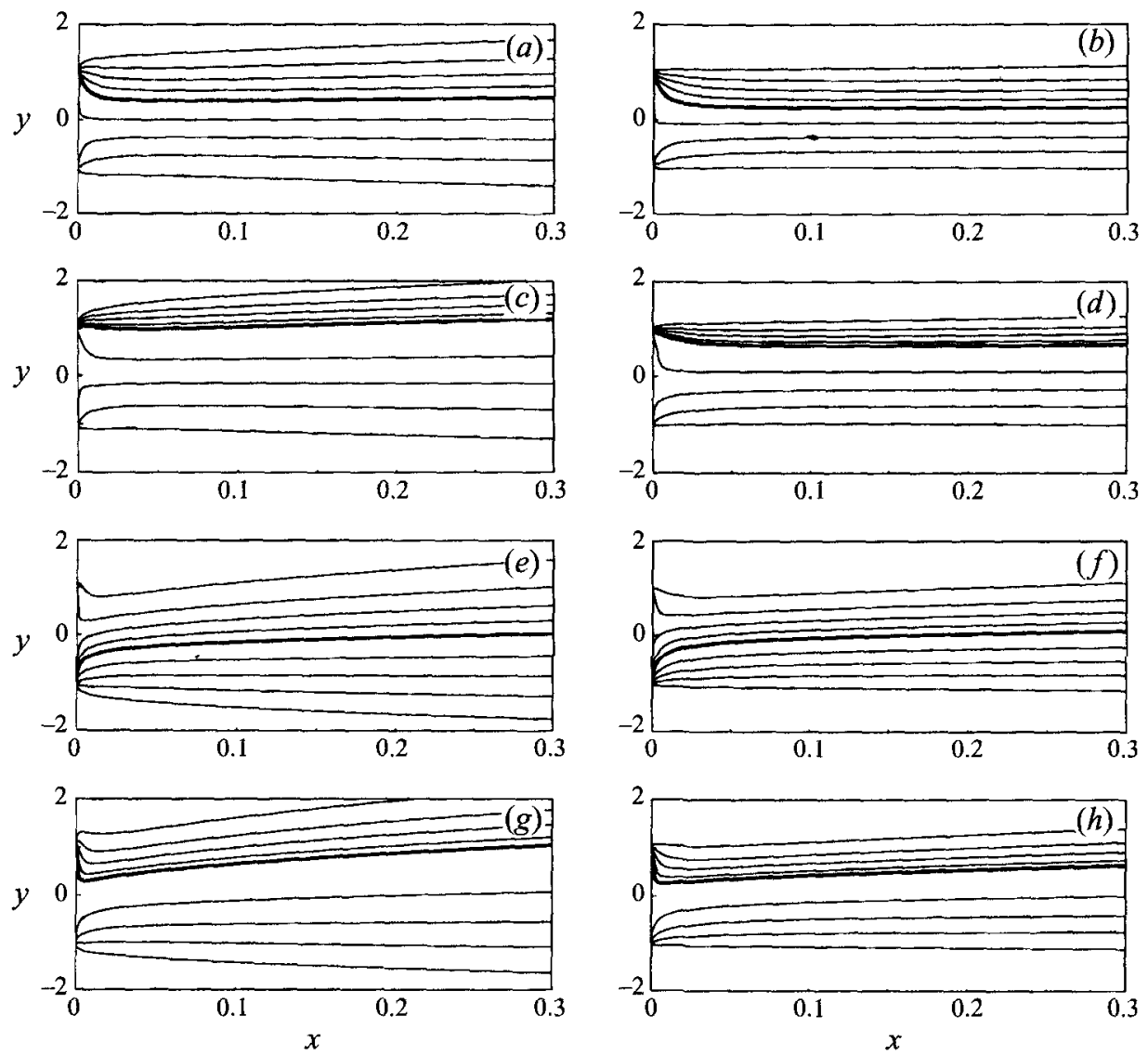

FIGURE 3. Isotherms for $\sigma=0, \operatorname{Pr}=1$ and the same values of $(\alpha, S, \gamma)$ as figure 2. Five equispaced contours between $T=1$ and $T=T_{e}$ are shown, with $T_{e}=2$ for the left-hand column and $T_{e}=7$ for the right-hand column.

change the origin of the recirculating fluid. Thus, in all the cases displayed in figure 2, the fluid recirculating on both sides of the wake comes from the oxygen stream and has already crossed the flame at some downstream position, whereas on the contrary, for sufficiently small Froude numbers, the fluid recirculating in the oxygen side comes from the fuel stream and crosses the flame only after reversing the sense of its motion for a second time.

Some pressure distributions are given in figure 4 . The pressure gradient is always adverse in the bulk of the recirculation region, pushing the recirculating fluid toward the wall against the viscous shear stresses of the outer streams. This avoids the premature termination of the recirculation region, which is not permitted by the conditions (2.11) of zero displacement until the viscosity has smoothed out the velocity profiles or the gas expansion due to the heat release has generated sufficient volume to fill the available space. Further downstream the pressure keeps growing if $\gamma$ is below a certain critical value $\gamma_{c}$, dependent on the other parameters, or attains a maximum somewhere behind the recirculation region if $\gamma$ is above $\gamma_{c}$. It is also worth noticing that the pressure is a decreasing function of $x$ in a very tiny region immediately behind the wall, not clearly visible in figure 4 but that can be seen in figure $1(a)$.

The flow for small values of $x$ consists of a recirculation region separated from 

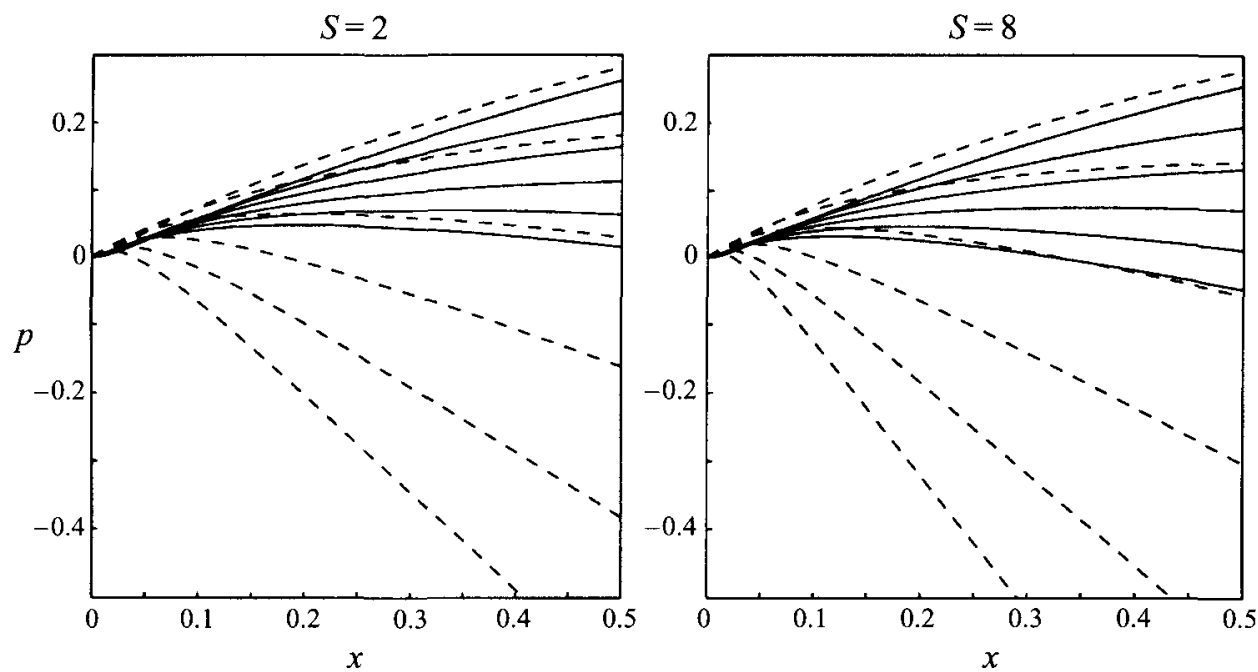

FIgURE 4. Pressure distributions for $\operatorname{Pr}=\alpha=1, S=2$ and 8 , and $\gamma=(1,2,3,4,5,6)$, from upper to lower curve in each family. Solid: $\sigma=0$; dashed: $\sigma=1$.

the outer uniform shear flows by two viscous shear layers issuing at the edges of the wall. Transport effects are negligible in the outer flows and in the recirculation region. In this last region the mixture fraction takes a uniform value $Z_{b}$, determined by the flow at larger distances downstream from the wall, and the motion of the fluid is induced by the entrainment of the shear layers. In terms of the stream function $\psi$, with $\rho u=\partial \psi / \partial y$ and $\rho v=-\partial \psi / \partial x$, the solution in these layers is of the form $\psi=$ $\pm\left[x^{2 / 3} f_{1}(\eta)+x f_{2}(\eta)+x^{4 / 3} f_{3}(\eta)+\cdots\right]$ and $Z=Z_{1}(\eta)+x^{1 / 3} Z_{2}(\eta)+x^{2 / 3} Z_{3}(\eta)+\cdots$, where $\eta=( \pm y-1) / x^{1 / 3}$ and the upper (resp. lower) signs correspond to the upper (lower) layer. The leading terms $f_{1}(\eta)$ and $Z_{1}(\eta)$ are the solution of a Goldstein (1930) self-similar problem, consisting of the momentum and mixture fraction conservation equations (the primes meaning differentiation with respect to $\eta$ )

$$
\left.\begin{array}{l}
{\left[T^{\sigma}\left(T f_{1}^{\prime}\right)^{\prime}\right]^{\prime}+\frac{2}{3} f\left(T f_{1}^{\prime}\right)^{\prime}-\frac{1}{3} T f_{1}^{\prime 2}=0,} \\
\left(T^{\sigma} Z_{1}^{\prime}\right)^{\prime}+\frac{2}{3} \operatorname{Prf}_{1} Z_{1}^{\prime}=0,
\end{array}\right\}
$$

with the boundary conditions

$$
f_{1}^{\prime}=0, \quad Z_{1}=Z_{b} \quad \text { for } \quad \eta \rightarrow-\infty,
$$

and

in the upper layer, or

$$
f_{1}=\alpha \eta^{2} / 2+o(1), \quad Z_{1}=0 \quad \text { for } \quad \eta \rightarrow \infty
$$

$$
f_{1}=\eta^{2} / 2+o(1), \quad Z_{1}=1 \quad \text { for } \quad \eta \rightarrow \infty
$$

in the lower layer, and $T\left(Z_{1}\right)$ given by (2.5).

Since the fluxes in the shear layers are proportional to $x^{2 / 3}$, the stream function in the recirculation region must be of the form $\psi=x^{2 / 3} F(y)+O(x)$, and the corresponding velocities lead to a pressure variation $p=-A x^{4 / 3}+\cdots$, which is too small to affect the flow in the shear layers to leading order. A pressure decreasing with $x$ is required for conservation of the total head on the streamline of the recirculating 


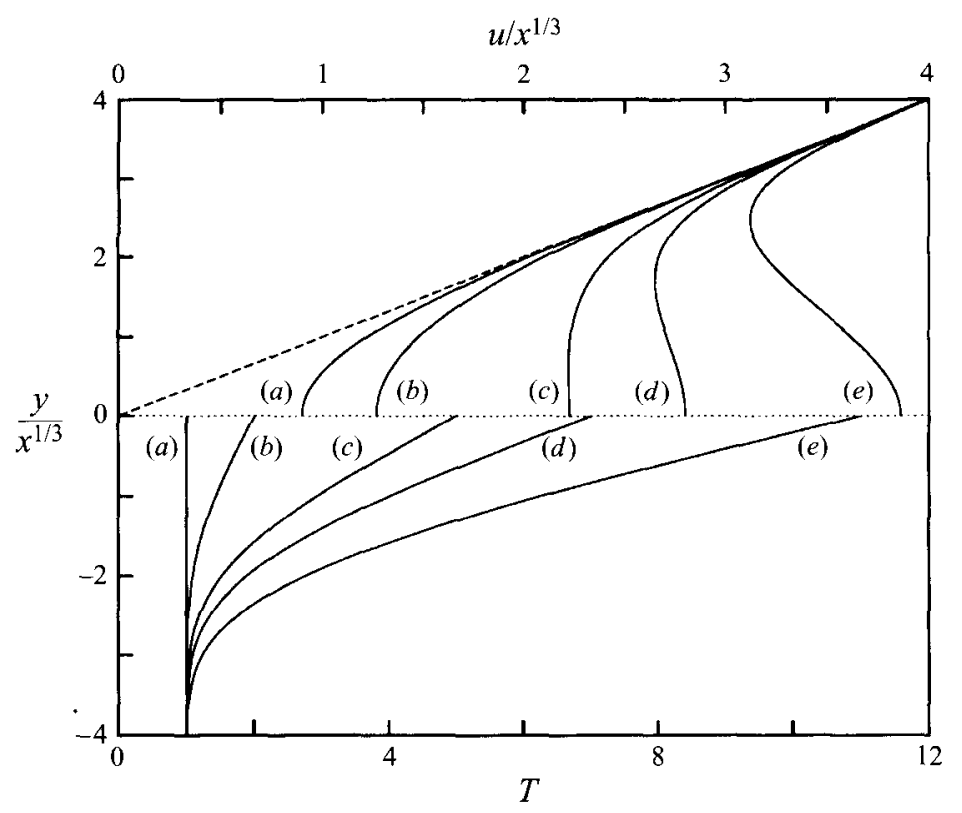

FIGURE 5. Self-similar velocity (upper part) and temperature (lower part) profiles for $\sigma=0$, $\operatorname{Pr}=S=\alpha=1$, and five values of $\gamma:(a) \gamma=0 ;(b) \gamma=1 ;(c) \gamma=4 ;(d) \gamma=6 ;(e) \gamma=10$.

flow that ends at the wall and separates the fluid ingested by each shear layer. The momentum equation (2.7) with $\rho=\rho_{b}=\rho\left(Z_{b}\right)$ becomes a second-order ordinary differential equation for the recirculating flow whose solution is

$$
F(y)=-\left(\frac{2 A}{\mathscr{A}}\right)^{1 / 2} \sin \left[\left(\rho_{b} \mathscr{A}\right)^{1 / 2}\left(y-y_{0}\right)\right],
$$

where $y_{0}$ and $\mathscr{A}$ are integration constants representing, respectively, the height of the dividing streamline and the ratio of the vorticity to the stream function. This ratio is a constant in the present solution, being determined by the flow in the bulk of the recirculation region. The values of $A$ and $y_{0}$ can be obtained from the conditions $F(1)=-f_{u}$ and $F(-1)=f_{l}$, expressing the matching of the recirculation region with the shear layers. Here $f_{u}$ and $f_{l}$ are the values of $f_{1}(-\infty)$ from the solutions of (3.1)-(3.3) in the upper and lower layers, respectively.

Once the leading-order solution is known, $f_{2}(\eta)$ and $f_{3}(\eta)$ can be found by solving linear equations with the boundary conditions $f_{2} \sim \pm F^{\prime}( \pm 1) \eta$ and $f_{3} \sim \pm F^{\prime \prime}( \pm 1) \eta^{2} / 2$ for $\eta \rightarrow-\infty$, and $\left(f_{2}, f_{3}+A / \alpha\right)$ or $\left(f_{2}, f_{3}+A\right)$ decaying exponentially to zero for $\eta \rightarrow \infty$. In particular, $f_{3}$ reflects the influence of the pressure variation on the shear layers, whereas this pressure variation leads to lateral velocities $\frac{4}{3}(A / \alpha) x^{1 / 3}$ and $-\frac{4}{3} A x^{1 / 3}$ in the outer flows of oxygen and fuel, respectively. The values of the constants $Z_{b}$ and $\mathscr{A}$ can in principle be extracted from the numerical solution of the full problem. Thus, for $\alpha=1$ and $\gamma=0$ the flow is symmetric and $Z_{b}=1 / 2$, whereas $\mathscr{A} \approx 3.9$, from graphs of $\omega / \psi$ near the solid.

The flow far downstream of the wall can also be described in a simple form, accounting for the change of sign of the pressure force at $\gamma=\gamma_{c}$. The solution for large values of $x$ does not depend on the thickness of the wall and, as described by Hakkinen \& Rott (1965) for a constant-density fluid, it consists of a self-similar viscous shear layer between two regions of uniform vorticity, with a pressure gradient 
such that these regions are not displaced by the presence of the viscous layer. For small values of $\gamma$ (in particular for $\gamma=0$ ) an adverse pressure gradient appears to balance the acceleration by viscous forces of the fluid near the solid subsequent to the release of the no-slip condition at $x=0$, which would displace the outer streams inward. On the other hand, this displacement would be outward for large values of $\gamma$, because the gas thermal expansion offsets the viscous acceleration, and therefore a favourable pressure gradient appears. The solution in the viscous layer is $\psi=x^{2 / 3} f_{\mathrm{HR}}\left(\eta_{\mathrm{HR}}\right), Z=Z_{\mathrm{HR}}\left(\eta_{\mathrm{HR}}\right), p=C x^{2 / 3}$, with $\eta_{\mathrm{HR}}=y / x^{1 / 3}$ and $f_{\mathrm{HR}}\left(\eta_{\mathrm{HR}}\right)$ and $Z_{H R}\left(\eta_{H R}\right)$ satisfying (3.1), with a term $2 C / 3$ added to the right-hand side of the first equation, and the boundary conditions

$$
\left.\begin{array}{lll}
f_{H R}^{\prime}=\alpha \eta_{H R}+o(1), \quad Z_{H R}=0 & \text { for } \quad \eta_{H R} \rightarrow \infty, \\
f_{H R}^{\prime}=-\eta_{H R}+o(1), \quad Z_{H R}=1 & \text { for } \quad \eta_{H R} \rightarrow-\infty .
\end{array}\right\}
$$

Sample values of $\gamma_{c}$ (at which $C=0$ ) from the solution of (3.1) and (3.5) for $\alpha=P r=1$ and $S=(1,2,4,8)$ are $\gamma_{c}=(1.665,1.617,1.496,1.358)$ when $\sigma=0$, and $\gamma_{c}=(1.241,1.214,1.142,1.052)$ when $\sigma=1$. Some velocity and temperature profiles are shown in figure 5. As can be seen, the favourable pressure gradient for $\gamma>\gamma_{c}$ has a strong effect on the light fluid surrounding the flame, leading to a local velocity maximum for sufficiently large values of $\gamma$.

The solution of (2.5)-(2.11) describes the flow behind the wall up to distances of order $\left(\delta / R e^{2 / 7} h\right)^{3}$ or $\left(\delta / R e^{1 / 4} h\right)^{3}$, for confined or unconfined flows respectively, in the present non-dimensional variables. Lateral displacements of the velocity profiles become acceptable at these distances, leading to the interaction regions mentioned in the previous section. Contrary to the case of a constant-density fluid first analysed by Stewartson (1969) and Messiter (1970) for an infinitely thin plate and by Vatsa, Werle \& Verdon $(1981,1982)$ for plates of finite thickness, the displacements due to the thermal expansion will be outward when $\gamma>\gamma_{c}$ (because a favourable pressure gradient was necessary to confine the flow nearer to the wall) and will induce adverse pressure gradients in the oncoming streams ahead of the edge of the wall. These gradients will reduce the skin friction seen by the near wake and determining the scales (2.2) of this region from its unperturbed value. Conceivably, the induced adverse pressure gradients could lead to separation on the wall for sufficiently large gas expansions, invalidating the previous results. Here the flow in the triple-deck interaction region of unconfined streams is briefly discussed, in an attempt to estimate a range of gas expansions over which separation does not occur. Referring the reader to the literature for more detailed accounts (e.g. Smith 1982), we notice that appropriate scaling factors for the variables $(x, y, u, v, p)$ in the lower deck are

$$
\frac{\rho_{\infty}^{1 / 4} U_{F}^{3 / 2}}{\mu_{\infty}^{1 / 4} \lambda_{F \infty}^{5 / 4}}, \quad \frac{\mu_{\infty}^{1 / 4} U_{F}^{1 / 2}}{\rho_{\infty}^{1 / 4} \lambda_{F \infty}^{3 / 4}}, \quad \frac{\mu_{\infty}^{1 / 4} \lambda_{F \infty}^{1 / 4} U_{F}^{1 / 2}}{\rho_{\infty}^{1 / 4}}, \quad \frac{\mu_{\infty}^{3 / 4} \lambda_{F \infty}^{3 / 4}}{\rho_{\infty}^{3 / 4} U_{F}^{1 / 2}}, \quad \rho_{\infty}^{1 / 2} \mu_{\infty}^{1 / 2} \lambda_{F \infty}^{1 / 2} U_{F},
$$

which take the place of the corresponding factors (2.2) used before. Here $\lambda_{F_{\infty}}=$ $-(\partial u / \partial y)_{y=0^{-}}$ahead of the interaction region, which differs from $\lambda_{F 0}$ in (2.2). Notice also that the wall appears as infinitely thin in the scale of this region. The distances, velocities and pressure variations scaled with the factors (3.6) will be denoted with a tilde. These variables still satisfy equations (2.6)-(2.9) on the two sides and downstream 

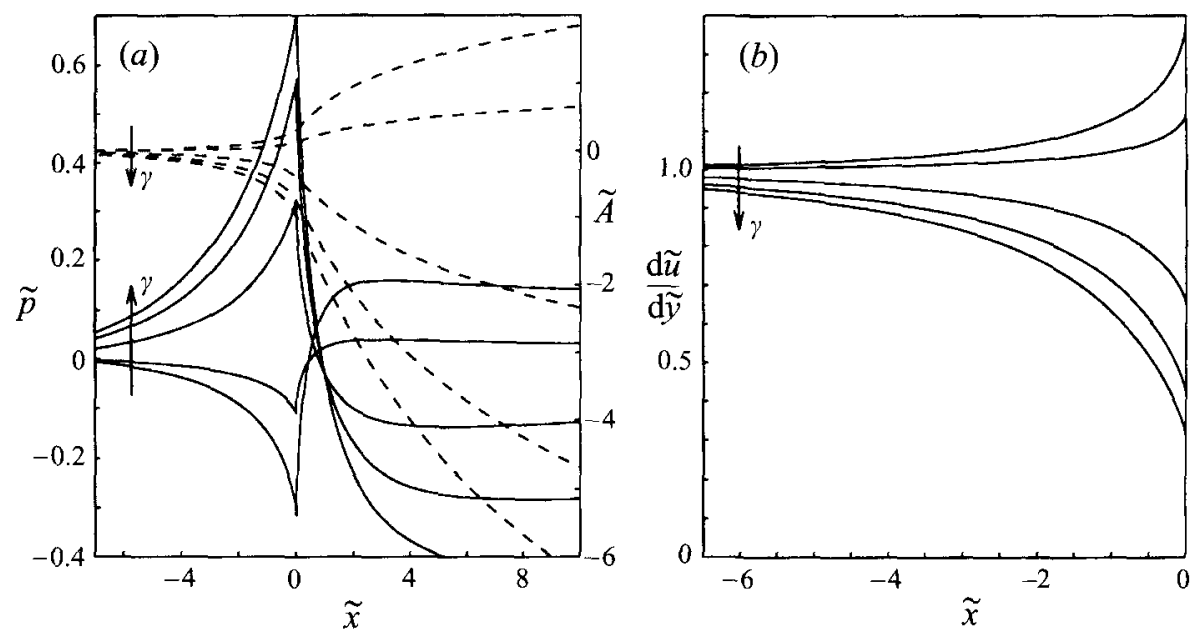

FIgURE 6. (a) Pressure (solid) and negative of the displacement (dashed), and (b) shear at the wall, for $\sigma=0, \operatorname{Pr}=S=\tilde{\alpha}=1$, and $\gamma=(0,1,5,10,15)$, increasing as indicated by the arrows.

of the wall, whereas the conditions (2.10) and (2.11) change to

$$
\left.\begin{array}{ll}
\tilde{u}=\tilde{\alpha}\left(\tilde{y}+\tilde{A}_{+}(\tilde{x})\right), \quad Z=0 & \text { for } \tilde{y} \rightarrow \infty \quad \text { and for } \tilde{x} \rightarrow-\infty, \tilde{y}>0, \\
\tilde{u}=-\tilde{y}+\tilde{A}_{-}(\tilde{x}), \quad Z=1 & \text { for } \tilde{y} \rightarrow-\infty \quad \text { and for } \tilde{x} \rightarrow-\infty, \tilde{y}<0, \\
\tilde{u}=\tilde{v}=\partial Z / \partial \tilde{y}=0 & \text { at } \tilde{y}=0^{ \pm}, \tilde{x}<0,
\end{array}\right\}
$$

where $\tilde{A}_{ \pm}(\tilde{x})$ are the unknown displacements of the boundary layers in the oxygen and fuel streams due to the interaction, counted positive inward, and $\tilde{\alpha}=\lambda_{o \infty} / \lambda_{F \infty}$. The pressure variations induced by such displacements in the corresponding streams are (Stewartson 1969)

$$
\tilde{p}_{+}(\tilde{x})=\frac{\beta}{\pi} \int_{-\infty}^{\infty} \frac{\mathrm{d} \tilde{A}_{+} / \mathrm{d} \xi}{\tilde{x}-\xi} \mathrm{d} \xi \quad \text { and } \quad \tilde{p}_{-}(\tilde{x})=\frac{1}{\pi} \int_{-\infty}^{\infty} \frac{\mathrm{d} \tilde{A}_{-} / \mathrm{d} \xi}{\tilde{x}-\xi} \mathrm{d} \xi,
$$

where $\beta=\left(U_{o} / U_{F}\right)^{2}$ and the principal values of the integrals are understood. These are the pressures driving the flow, and must satisfy

$$
\tilde{p}_{+}=\tilde{p}_{-} \quad \text { for } \quad \tilde{x}>0 \text {. }
$$

The distributions of pressure and non-dimensional skin friction obtained from the numerical solution of (2.5)-(2.9) and (3.7)-(3.9) in the symmetric case $\tilde{\alpha}=S=1$ are given in figure 6 for $\sigma=0, \operatorname{Pr}=1$, and several values of $\gamma$. As can be seen, the pressure gradient on the wall changes from favourable to adverse, and the skin friction changes from increasing to decreasing, for $\gamma$ about the correct critical value $\left(\gamma_{c} \approx 1.665\right.$ for the present values of the parameters; the pressure should be uniform throughout the flow for $\gamma=\gamma_{c}$ ). The skin friction remains positive even for the largest values of $\gamma$ used in these computations. Further computations with $0<\sigma \leqslant 1$ show that the minimum skin friction, always attained at the edge of the wall, decreases with increasing $\sigma$ but does not vanishes until $\gamma$ is well above 10. Thus separation, with its important consequences for flame stabilization, seems to be unlikely for the values of $\gamma$ typical of the most exothermic reactions found in combustion processes, which are in the range 5-7, and these results, limited as they are, provide evidence that the solution obtained in the near-wake region on the assumption that no separation occurs upstream is applicable under realistic conditions. 


\section{Asymptotic limit of very exothermic reactions}

In this section the structure of the solution of (2.5)-(2.11) in the asymptotic limit $\gamma \rightarrow \infty$, corresponding to very exothermic chemical reactions, is discussed. A limitation to the validity of this asymptotic analysis arises from the outset, because if separation due to the gas expansion occurs in the interaction region before the end of the wall for a certain value of $\gamma$, the problem (2.5)-(2.11) is not relevant for $\gamma$ above this value. The results at the end of the previous section, however, show that boundary layer separation requires very large values of $\gamma$, while the asymptotic results of this section provide a good description of the flow even for moderate values of this parameter, in the range of practical interest for gas-phase combustion. This fortunate circumstance renders the asymptotic results useful despite their formal limitation. Moreover, at least one case exists in which symmetry conditions prevent any outward displacement and no pressure perturbation appears upstream for any $\gamma \ll \boldsymbol{R}^{1 /(2 \sigma+1)}$. Such conditions could be realized by setting up a periodic array of alternating streams of fuel and oxygen in adjacent channels formed by an infinite sequence of equal parallel walls like the one considered here, simulating an array of injectors.

Let us begin by describing Goldstein's shear layers for small values of $x$. Here the upper layer is discussed, the other one being analogous, and for convenience $\alpha=1$ is set in $(3.3 a)$ using the invariance of (3.1) under scale transformations. Since the transition from the cold reactant stream to the hot recirculating fluid occurs across this layer, the whole range of temperatures, from 1 to $O(\gamma)$, is present. In addition, in the absence of pressure forces the fluid expands freely toward the recirculation region on receiving heat, and the high-temperature region of the shear layer is very thick. As can be easily verified, eqs. (3.1) have solutions with temperature of order unity for $\eta=O(1)$ and growing potentially for $(-\eta) \gg 1$, of the form $f_{1} \sim b /(-\eta)^{1-\sigma m}$ and $T \sim a(-\eta)^{m}$, for which

$$
\frac{u}{x^{1 / 3}}=T f_{1}^{\prime} \sim(1-\sigma m) \frac{a b}{(-\eta)^{2-(1+\sigma) m}}
$$

Here $a$ is a free constant, $b=3[(1+\sigma) m-1] a^{\sigma} /(2 P r)$, and

$$
m(\sigma, \operatorname{Pr})=\frac{\left[k^{2}+12(4 P r-1) j\right]^{1 / 2}-k}{2 j}
$$

with

$$
j=(1+\sigma)[2+\sigma-2 \operatorname{Pr}(1+2 \sigma)] \quad \text { and } \quad k=2 \operatorname{Pr}(5+7 \sigma)-5-4 \sigma,
$$

satisfies $1<m<2 /(1+\sigma)$ for $0 \leqslant \sigma<1$ and any $P r$. For these solutions the temperature reaches values of order $\gamma$ when $(-\eta)$ becomes of order $\eta_{i}=\gamma^{1 / m} \gg 1$, and then $f_{1}$ is of order $f_{i}=1 / \gamma^{1 / m-\sigma} \ll 1$ and the scaled velocity is $T f_{1}^{\prime}=O\left(1 / \gamma^{2 / m-1+\sigma}\right) \ll$ 1. Thus the flux and the velocity are very small in the region of high temperature, despite its thickness. Appropriate variables to describe this region are $f_{h}=f_{1} / f_{i}$, $T_{h}=T / \gamma$ and $\eta_{h}=\eta / \eta_{i}$, and the relationship between the temperature and the mixture fraction in (2.5) becomes

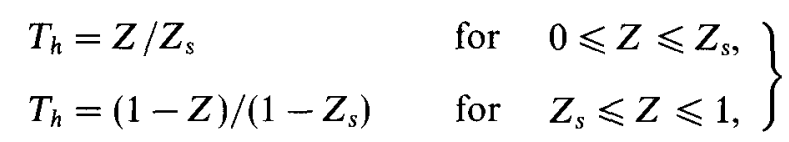

to leading order. Equations (3.1) are left invariant by this rescaling, and must be 
solved with the boundary conditions (3.2) and the matching conditions

$$
f_{h} \sim \frac{b}{\left(-\eta_{h}\right)^{1-\sigma m}}+\cdots, \quad T_{h} \sim a\left(-\eta_{h}\right)^{m}+\cdots \quad \text { for } \quad\left(-\eta_{h}\right) \rightarrow 0,
$$

where further terms of the expansions, involving two other free constants in addition to $a$, can be computed from (3.1). In particular, $m=9 / 5$ and $b=6 / 5$ for $\sigma=0, \operatorname{Pr}=1$. For $\sigma=1$, (4.2) gives $m=1$, but the asymptotic expansion for $\left(-\eta_{h}\right) \ll 1$ contains in this case logarithms of $\left(-\eta_{h}\right)$. The solution of (3.1), (3.2) and (4.4) depends on $\sigma, \operatorname{Pr}$, $Z_{b}$, and also on $Z_{s}$ if the shear layer contains the flame $\left(Z_{b}>Z_{s}\right)$. This solution is easy to obtain numerically using the two-parameter group of transformations that leave (3.1) invariant, and determines $a$ (which comes out proportional to $Z_{b}$ if $Z_{b}<Z_{s}$ )

Another structure of interest arises when one of the reactants is at rest, typically the oxygen, corresponding to $\alpha=0$ in the formulation of $\S 2$. Then there is no possibility of generating appreciable pressure gradients and the flow consists only of the Navier-Stokes region about the lower edge of the wall followed by a Goldstein's shear layer between the fuel stream and the oxygen mixture. In the limit $\gamma \rightarrow \infty$ the asymptotic structure of this layer is similar to the one just discussed if the oxygen temperature is high, with this reactant playing then the role of the recirculating fluid. However, if both reactants are cold, the structure of the shear layer near the oxygen side is different and depends strongly on the Prandtl number. With $\sigma=0$ and the variables scaled as before, the solution for $\operatorname{Pr}<1$ is of the form $f_{h} \sim f_{\infty}+b \exp \left(-\frac{2}{3} f_{\infty}(1-P r) \eta_{h}\right)$ and $T_{h} \sim a \exp \left(-\frac{2}{3} f_{\infty} \operatorname{Pr} \eta_{h}\right)$, with $f_{\infty}>0$, for $\eta_{h} \rightarrow \infty$, whereas for $\operatorname{Pr}>1$ the high temperature region extends only to a finite $\eta_{h}=\eta_{h_{u}}$, the solution being of the form $f_{h} \sim(3 / 2)\left(m^{\prime}-1\right) /\left[\operatorname{Pr}\left(\eta_{h_{u}}-\eta_{h}\right)\right]$ and $T_{h} \sim a\left(\eta_{h_{u}}-\eta_{h}\right)^{m^{\prime}}$, with $m^{\prime}=3(4 \mathrm{Pr}-1) /[2 m(\operatorname{Pr}-1)]>3$, for $\left(\eta_{h_{u}}-\eta_{h}\right) \ll 1$. The differences arise because the effect of the heat conduction extends farther than that of the viscosity when $\operatorname{Pr}<1$, preheating the oxygen mixture and decreasing its density before it is ingested by the shear layer, which results in a finite ingestion rate from this side of the layer. On the other hand, for $\mathrm{Pr}>1$ the viscosity reaches farther than the heat conduction and the ingested fluid is denser, resulting in an infinite ingestion rate with the scales of the high-temperature region.

Coming back to the case $\alpha>0$, let us consider the recirculating flow between the two shear layers. The ingestion of the layers induces velocities of order $\gamma^{1+\sigma-1 / m} x^{2 / 3}$, and the pressure variations are of order $\gamma^{1+2 \sigma-2 / m} x^{4 / 3}$ for sufficiently small $x$. As was mentioned before, these pressure variations are too weak to affect the cold streams of the reactants, which therefore do not suffer any lateral displacement, confining the growth of the shear layers to the space behind the wall. In these conditions, the high-temperature regions of the two shear layers interfere, and Goldstein's self-similar solution ceases to be applicable, at a distance of order $x_{b}=\eta_{c}^{-3}=\gamma^{-3 / m} \ll 1$ behind the wall, obtained by writing $|y-1|=1$ in the expression for $\eta$. This $x_{b}$ is the characteristic length of the recirculation region, where the velocity of the fluid is of order $u_{b}=\gamma^{1+\sigma-3 / m}$ and the pressure variations are of order $p_{b}=\gamma^{1+2 \sigma-6 / m}$. Since such pressure variations are still too small to affect the flow in the cold regions of the shear layers when $\sigma<1$, that flow remains self-similar over the recirculation region and beyond. For example, for $\sigma=0$ and $\operatorname{Pr}=1, x_{b}=\gamma^{-5 / 3}, u_{b}=\gamma^{-2 / 3}$ and $p_{b}=\gamma^{-7 / 3}$, while the velocity and the dynamic pressure in the cold parts of the shear layers are much larger, of orders $\gamma^{-5 / 9}$ and $\gamma^{-10 / 9}$. For $\sigma=1, x_{b}=\gamma^{-3}$ and the velocities in the hot and cold fluids are both of order $\gamma^{-1}$. Logarithmic plots of the distance from the wall to the farthest point of negative velocity as a function of $\gamma$, obtained from the 


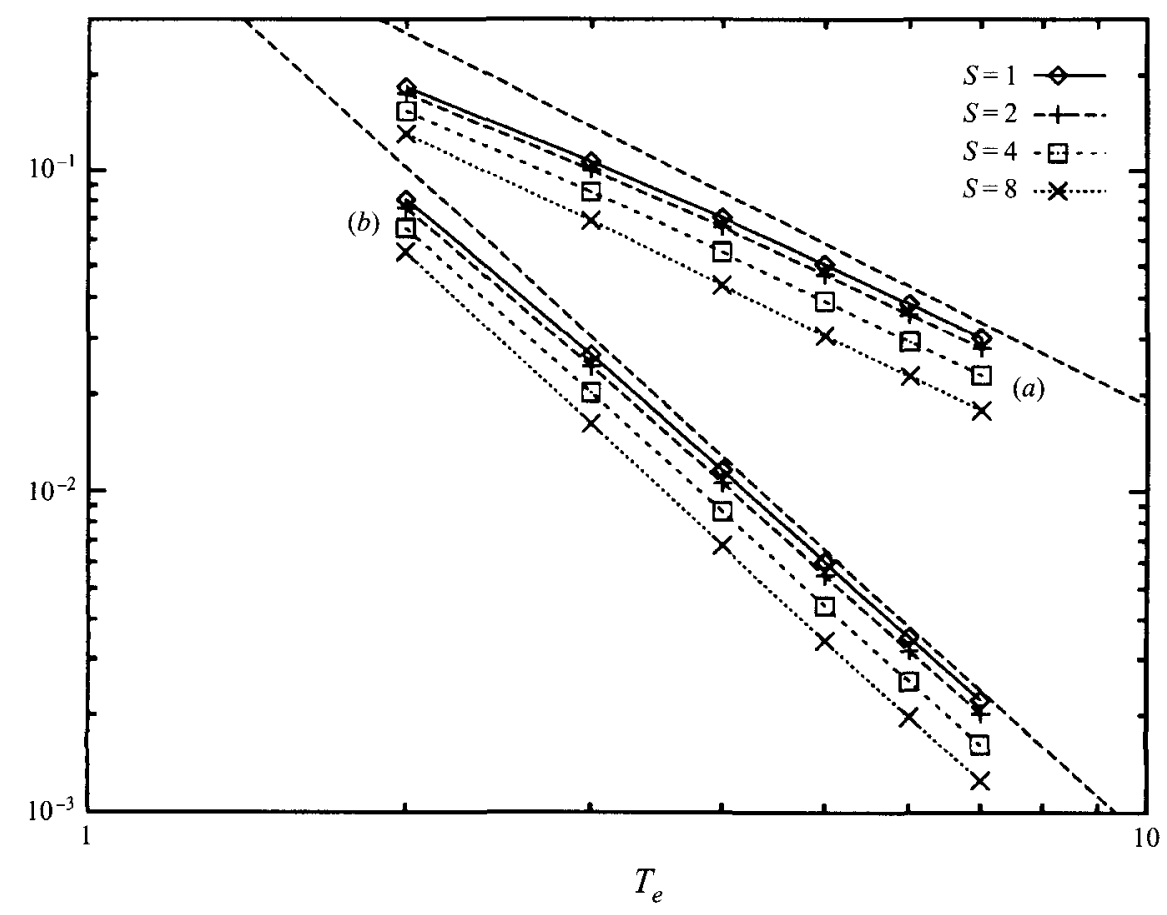

FIGURE 7. Distance to the farthest point with negative velocity as a function of $T_{e}=1+\gamma$ for $\operatorname{Pr}=\alpha=1$ and $(a) \sigma=0,(b) \sigma=1$. The longer dashed lines have the slopes $(a)-5 / 3,(b)-3$ predicted by the asymptotic theory.

numerical solution of (2.5)-(2.11), are given in figure 7 for these two cases, showing a rapid approach to the asymptotic behaviour as $\gamma$ increases.

For $x \gg x_{b}$ the region of high temperature is confined by non-compliant streams of heavy fluids and cannot keep on growing as in the freely expanding shear layers for $x \ll x_{b}$. Since heat conduction from the flame continually heats up more fluid, its motion in the strip $|y| \leqslant 1$ requires a favourable pressure gradient, whose origin is in minute deflections of the outer streams, to be discussed later. In the strip of high temperature, the balance of convection, $O\left(u^{2} / \gamma x\right)$, viscous force, $O\left(\gamma^{\sigma} u\right)$, and pressure force, gives $u=O\left(\gamma^{1+\sigma} x\right)$ and $\Delta p=O\left(\gamma^{1+2 \sigma} x^{2}\right)$, and, under the action of the gradient of this pressure, the velocity profile of the light fluid rapidly changes from the wake-like shape prevailing for $x \ll x_{b}$ to the jet-like shape depicted in figure 5 . Eventually the pressure variations become of order unity, for $x$ of order $x_{c}=1 / \gamma^{\sigma+1 / 2}$, and the strip of light fluid begins to thicken, displacing the cold streams sideways.

The solution in the high-temperature region for $x_{b} \ll x \ll x_{c}$ is of the form

$$
\psi=\gamma^{\sigma} x G(y), \quad Z=Z(y), \quad p=p_{0}-\gamma^{1+2 \sigma} x^{2} B / 2,
$$

where $G(y)$ and $Z(y)$ satisfy the equations (primes meaning now derivatives with respect to $y$ )

$$
\left[T_{h}^{\sigma}\left(T_{h} G^{\prime}\right)^{\prime}\right]^{\prime}+G\left(T_{h} G^{\prime}\right)^{\prime}-T_{h} G^{2}=-B \quad \text { and } \quad\left(T_{h}^{\sigma} Z^{\prime}\right)^{\prime}+\operatorname{Pr} G Z^{\prime}=0
$$


with the scaled temperature given by (4.3), and the boundary conditions

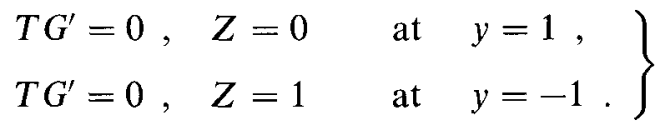

The conditions of zero velocity at the boundaries express the matching of this region to others where much heavier fluid under the same pressure gradient moves much slower. For sufficiently small values of $x$ the strip of light fluid is limited by warm strata of Goldstein's shear layer, where the velocity is given by (4.1) and $\rho=\left[a(-\eta)^{m}\right]^{-1}$. Using these quantities, the condition $\rho u^{2}=O(\Delta p)$ yields $(-\eta)=O\left[x \gamma^{3(1+2 \sigma) / 4}\right]^{-4 / 3(4-(1+2 \sigma) m)}$ for the inner boundary of the region of the shear layer not affected by the pressure gradient at a given $x$. This $(-\eta)$ becomes of order unity for $x$ of order $\gamma^{-3(1+2 \sigma) / 4}$, and no remnant of Goldstein's self-similar flow is left for larger values of $x$.

It turns out that (4.6) and (4.7) determine not only $G(y)$ and $Z(y)$ but also the unknown constant $B$ in the expression for the pressure. This is because any solution of (4.6) satisfying the four boundary conditions (4.7) also satisfies the two additional conditions

$$
\int_{-1}^{1} T_{h} G^{2} \mathrm{~d} y=2 B \quad \text { and } \quad \int_{-1}^{1} G Z^{\prime} \mathrm{d} y=0,
$$

expressing the conservation of the fluxes of momentum plus pressure and mixture fraction in the strip. Conditions (4.8) are obtained by integrating equations (4.6) across the strip and using the asymptotic expansions of the solution near the boundaries. Near the upper boundary, $(1-y) \ll 1$, these expansions are of the form

$$
G=\frac{d_{0} B^{\sigma /(1+2 \sigma)}}{(1-y)^{(1-2 \sigma) /(1+2 \sigma)}}+\cdots, \quad T_{h}=c_{0} B^{1 /(1+2 \sigma)}(1-y)^{4 /(1+2 \sigma)}+\cdots
$$

if $\sigma<1 / 2$, where $c_{0}$ and $d_{0}$ are known functions of $\sigma$ and $\operatorname{Pr}$, or

$$
\left.\begin{array}{l}
G=\frac{c^{\sigma}}{\sigma P r}+d(1-y)^{1 / \sigma P r-1 / \sigma+1}+\frac{\sigma^{2}}{2 \sigma-1} \frac{P r}{\sigma P r-1} \frac{B}{c^{\sigma+1}}(1-y)^{2-1 / \sigma}+\cdots \\
T_{h}=c(1-y)^{1 / \sigma}+\cdots
\end{array}\right\}
$$

if $\sigma>1 / 2$, where $c$ and $d$ are free constants, and similar expressions hold for $(1+y) \ll 1$. Because of $(4.9 a)$, the diffusion fluxes of momentum and mixture fraction and the convective flux of momentum are zero across the lateral boundaries.

Numerical integration of (4.6) was carried out with a shooting method using the previous expansions (with two more terms of the expansions $(4.9 a)$ providing two free constants, much as in (4.4) above). Some results for $\sigma=0$ and $\operatorname{Pr}=1$ are $B=(56.69,61.11,74.35,96.25)$ for $S=(1,2,4,8)$.

As was already mentioned in the paragraph above (4.5), the pressure variations become of order unity and the strip of hot fluid begins to open up when $x$ becomes of order $x_{c}=1 / \gamma^{\sigma+1 / 2}$. By then (4.5) gives velocities of order $\gamma^{1 / 2}$ and the flow in the strip is described by equations (2.5)-(2.9) rewritten in terms of the scaled variables $X=x / x_{c}, u_{h}=u / \gamma^{1 / 2}, v_{h}=v / \gamma^{1+\sigma}, T_{h}=T / \gamma$ and $\rho_{h}=\gamma \rho$, of order unity here, with the boundary conditions

$$
\left.\begin{array}{ccc}
u_{h}=Z=0 & \text { at } & y=\delta_{+}(X) \\
u_{h}=Z-1=0 & \text { at } & y=\delta_{-}(X)
\end{array}\right\}
$$


and

$$
u_{h} \rightarrow X G^{\prime}(y), \quad Z \rightarrow Z(y) \quad \text { given by (4.6) and (4.7), for } X \rightarrow 0 .
$$

Here $\delta_{ \pm}$are the unknown upper and lower boundaries of the strip. The solution near these boundaries is still of the form $(4.9 a)$, with $(1-y)$ replaced by $\left|\delta_{ \pm}-y\right|$ and the coefficients of the expansions depending on $X$. As before, the fluxes of momentum and mixture fraction are conserved, leading to

$$
\frac{\mathrm{d}}{\mathrm{d} X} \int_{\delta_{-}}^{\delta_{+}} \rho_{h} u_{h}^{2} \mathrm{~d} y+\left(\delta_{+}-\delta_{-}\right) \frac{\mathrm{d} p}{\mathrm{~d} X}=0 \quad \text { and } \quad \frac{\mathrm{d}}{\mathrm{d} X} \int_{\delta_{-}}^{\delta_{+}} \rho_{h} u_{h} Z \mathrm{~d} y=0,
$$

which would permit solving the problem if $\delta_{ \pm}(X)$ were known. Two further relations between $\delta_{ \pm}(X)$ and $p(X)$, closing the problem, arise from the analysis of the cold streams. There the effect of the viscosity is negligible and the vorticity remains uniform in each stream, except in two thin layers of thickness $O\left(x_{c}^{1 / 2}\right)$ around the strip that need not be considered in detail. The velocity profiles are linear and the conditions (2.11) of zero displacement of these profiles are satisfied at infinity only if they are satisfied everywhere else; i.e. the outward displacement of each streamline in the lower stream must equal the increase of the velocity on it, and the outward displacement of each streamline in the upper stream must equal $\alpha^{-1}$ times the increase of outward velocity on it. In addition, because of the low density, the mass flux across the strip is only of order $\gamma^{-1 / 2}$, much smaller than the fluxes of the cold streams over regions of comparable thickness, in such a way that the boundaries of the strip appear as impermeable from the point of view of the outer streams. Bernoulli's theorem on the streamlines bounding the region of high temperature above and below then yields

$$
p+\frac{1}{2} \alpha^{2}\left(\delta_{+}-1\right)^{2}=p+\frac{1}{2}\left(\delta_{-}+1\right)^{2}=0,
$$

which are the two relations sought for.

For large values of $X$ the upstream thickness of the strip becomes irrelevant and the flow takes on a self-similar form corresponding to the asymptotic limit of the Hakkinen-Rott solution for $\gamma \rightarrow \infty$, in which $\Psi=X^{2 / 3} f_{H R}\left(\eta_{H R}\right), Z=Z_{H R}\left(\eta_{H R}\right)$ and $p=C X^{2 / 3}(\Psi$ being the scaled stream function and $C<0)$, with $\eta_{H R}=y / X^{1 / 3}$, $\delta_{ \pm}=\Delta_{ \pm} X^{1 / 3}$ and $\alpha \Delta_{+}=-\Delta_{-}=(-2 C)^{1 / 2}$. The functions $f_{H R}\left(\dot{\eta}_{H R}\right)$ and $Z_{H R}\left(\eta_{H R}\right)$ satisfy (3.1), with the term $2 C / 3$ added to the right-hand side of the first of these equations, and the boundary conditions

$$
T_{h} f_{H R}^{\prime}=Z_{H R}=0 \text { at } \eta_{H R}=\Delta_{+} \quad \text { and } \quad T_{h} f_{H R}^{\prime}=Z_{H R}-1=0 \text { at } \eta_{H R}=\Delta_{-} .
$$

Near the upper and lower boundaries the stream function and the temperature are of the forms (4.9), with $-C$ taking the place of $B$ and extra factors of $3 / 2$ multiplying and dividing respectively the first and third terms of the first equation $(4.9 \mathrm{~b})$. The solution also determines $C$. For example $C \approx-2.365$ for the symmetric case $S=\alpha=1$ with $\sigma=0$ and $\operatorname{Pr}=1$.

To close this section the asymptotic limit $S \rightarrow \infty$, which is of interest because large values of $S$ are frequently found in practical cases, is briefly discussed. Unfortunately the asymptotic expansion of the solution in this limit is in powers of the logarithm of $S$, being therefore of limited applicability, so only the main features of this solution will be mentioned here. In the (upper) Goldstein layer for $x \ll x_{b}$ the thickness of the high-temperature region becomes very large below the flame, $O\left[(\ln S)^{1 / 3}\right]$ for $\sigma=0$ and $\operatorname{Pr}=1$, and the distance from the flame to the upper boundary of the layer becomes very small, $O\left[(\ln S)^{-2 / 3}\right]$. The scaled mass flux is $f_{h}^{\prime}=O\left[(\ln S)^{4 / 3}\right]$ 
above the flame and much smaller, $O\left[(\ln S)^{1 / 3}\right]$, underneath, and, since $f_{h}^{\prime}$ must be continuous, the velocity at the flame is effectively zero from the point of view of the region above it. This situation changes somewhat farther downstream, owing to the favourable pressure gradient. Thus, in the Hakkinen-Rott solution, the asymptotic analysis yields $C \approx-\left(\frac{3}{4} \ln S\right)^{-2 / 3}$, whereas the thickness of the hot region is still $O\left[(\ln S)^{1 / 3}\right]$ below the flame and $O\left[(\ln S)^{-1 / 6}\right]$ above the flame, and $f_{h}^{\prime}=O\left[(\ln S)^{4 / 3}\right]$ at both sides of the flame, which appears as a surface of zero shear from the point of view of the region above it.

\section{Flame attachment}

\subsection{Effect of the wall thickness}

The analysis of the previous sections was aimed at describing the flow field of an existing flame, and was carried out on the assumption that the chemical reaction is sufficiently fast for the flame to remain attached under any condition. The results, however, shed some light on the issue of conditions for flame attachment and the different types of flame lift-off, and will be discussed here in that connection.

Notice first that the local burning rate at each point of the flame is given by the diffusion fluxes of fuel and oxygen, which arrive in stoichiometric proportions from opposite sides of the flame. In a real flame, however, finite-rate chemistry effects, not accounted for in the present analysis, impose an upper limit on the admissible fluxes, because local extinction occurs when the diffusion time becomes shorter than the chemical time $t_{c h}=\rho_{F} / w_{c}$, where $w_{c}$ is the characteristic value of $w$ in the right-hand side of (2.1) evaluated at the adiabatic flame temperature (Liñan 1974).

Both the reciprocal of the diffusion time and the burning rate are conveniently measured by the local value of the scalar dissipation $\chi=(\mu / \rho P r)(\partial Z / \partial y)^{2}$ at the flame. Maps of the scalar dissipation $\chi$ with the flame superimposed are given in figure 8 for the same sets of parameter values used in figures 2 and 3 . In the present non-dimensional variables $\chi$ tends to infinity at the edges of the wall (see §5.2), but away from these points the scalar dissipation on the flame, and therefore the possibility of local extinction, depends strongly on the values of the parameters. For $\alpha=1$, the flame with $S=2,(a)$ and $(b)$, manages to avoid the region of higher dissipation until very near the wall, whereas the flame with $S=8,(c)$ and $(d)$, lies in the middle of that region (which anyway is shorter than for $S=2$ ). For $\alpha=0.2$, the flame with $S=2,(e)$ and $(f)$, starts at the lower (fuel) edge, where the dissipation is higher, but now lies on top of the ridge of maximum dissipation, and the flame with $S=8,(g)$ and $(h)$, starts at the upper (air) edge and immediately bends downward.

Upon inspection of figures 2 and 8 two possibilities present themselves.

In cases like $(a)$ and $(b)(\alpha=1, S=2)$ or $(g)$ and $(h)(\alpha=0.2, S=8)$, and to a lesser extent also in cases $(e)$ and $(f)(\alpha=0.2, S=2)$, the velocity of the flow on the flame is directed toward the wall when the flame enters the region of highest dissipation. This region can then be considered as the flame tail rather than the flame head, in the sense that if the flame extinguishes here it can still survive downstream, where $\chi$ is smaller. Of course the flow between the extinction point and the wall will be different from the one described before, because the temperature on the surface $Z=Z_{s}$ will be lower than the adiabatic flame temperature and the reactants will diffuse into each other. These changes, however, need not have a strong effect on the flame downstream if the distance from the extinction point to the wall is small compared with the size of the recirculation region (as seems to be often the case, 

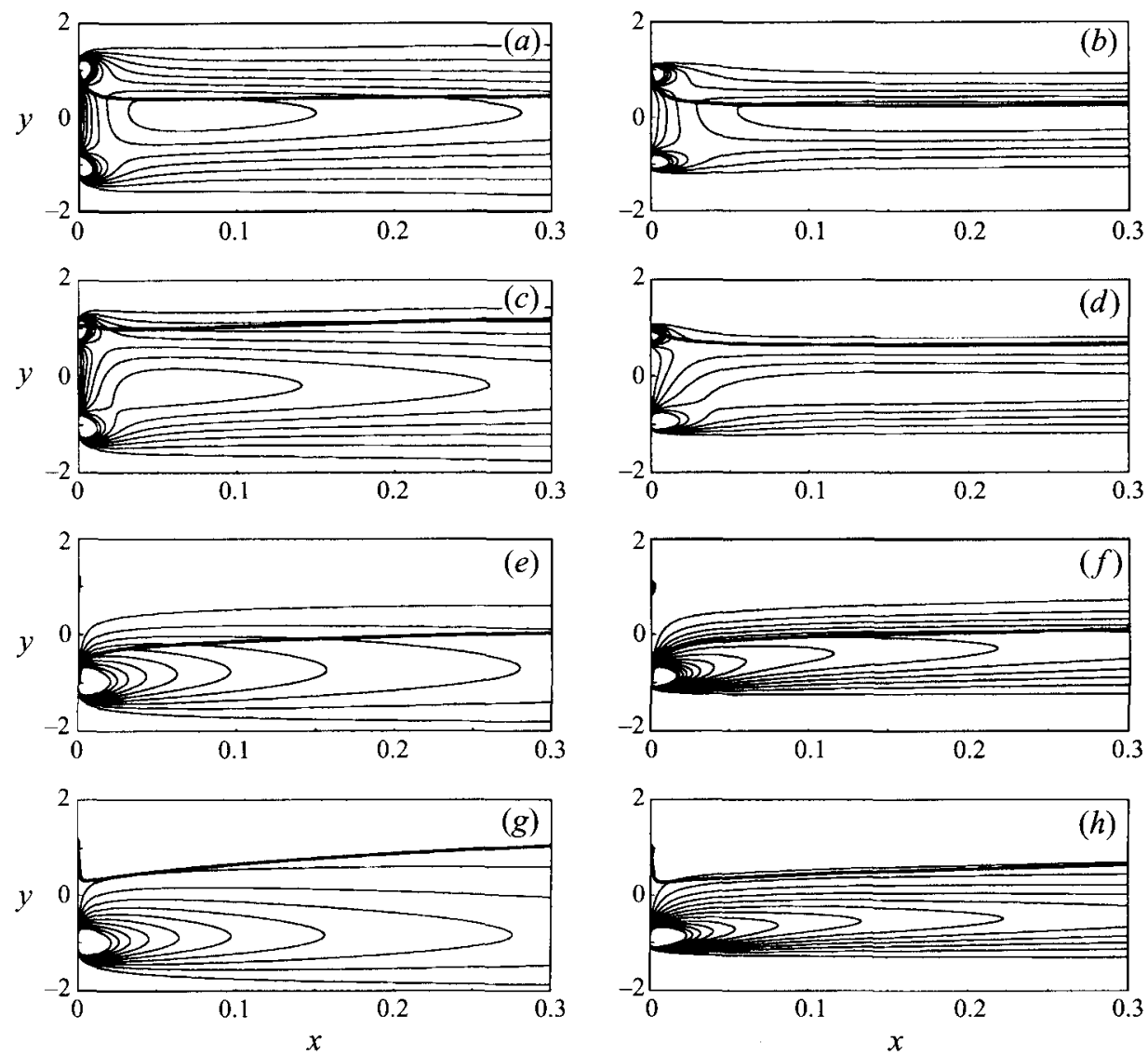

FIgURE 8. Scalar dissipation and flame for the cases of figure 2. Contour spacing is 0.03 in $(a)$ and $(c), 0.06$ in $(e)$ and $(g)$, and 0.2 in $(b),(d),(f)$ and $(h)$.

judging by the limited extent of the region of high dissipation), because the warm fluid still recirculates and the oxygen-fuel mixture, which will burn anyway on reaching the flame again, occupies only a small region. When, by changing the parameters, the tail of the flame reaches the rear or the lateral boundaries of the region of negative velocity, lift-off would occur in the fashion of the Type III instability of Takahashi \& Schmoll (1990).

On the other hand, in cases like $(c)$ and $(d)(\alpha=1, S=8)$ the flow is directed away from the wall over the region of the flame more prone to extinction. Now, if the nose of the flame (a triple flame; see Liñan 1994) cannot attach in a short region by the edge of the wall whose size will be estimated in the following subsection, it will not be able to exist downstream (though a diffusion flame could), and combustion will not occur at all in the vicinity of the injector. It should be noted here that the mere recirculation of hot fluid does not help in anchoring the flame front when the Lewis numbers are equal to unity, because the increase of temperature is associated with a decrease of the reactant concentrations, in such a way that the enthalpy (thermal plus formation) of the recirculating fluid is the same as in the cold streams. It should also be noted that, even under these adverse conditions, the region of low velocity around the rear stagnation point still provides a favourable environment for a steady triple flame to exist, with the two reactants and the combustion products coexisting all 
over the bubble. This possibility, however, falls outside the framework of the present analysis.

Quite independently of these results, it may also be remarked that inflexional velocity profiles are a characteristic feature of very exothermic reactions. Though no stability analysis will be carried out here, it seems a reasonable conjecture that such profiles may become unstable and lead to high turbulent strain rates that could extinguish the flame at some distance downstream of the recirculation region. This extinction would be in line with the observations of split flames by Vranos et al. (1968) and by others, and would occur in a region whose width bears no relation to the width of the fuel jet.

\subsection{Region of flame attachment}

The highest values of $\chi$, of order $t_{d}^{-1}=\lambda_{F 0}$ in dimensional variables, occur in the small Navier-Stokes regions of size $\delta_{N S}=R e^{-1 / 2} \delta=\left(\mu_{\infty} / \rho_{\infty} \lambda_{F 0}\right)^{1 / 2}$ around the edges of the wall, where the velocity of the fluid is of order $u_{N s}=\left(\mu_{\infty} \lambda_{F 0} / \rho_{\infty}\right)^{1 / 2}$ and the heat conduction toward the solid against the stream is important.

These small regions, which will not be analysed here, are known to play a key role in the attachment of flames with finite-rate chemistry when one of the reactants is at rest or when the thickness of the wall is comparable to $\delta_{N s}$. Then, a rough criterion of flame attachment (Lewis \& von Elbe 1961) is that the diffusion time $t_{d}$ should be of the order of the chemical time $t_{c h}$ or larger or, equivalently, that the propagation velocity of the corresponding stoichiometric premixed flame, $O\left(\mu_{\infty} / \rho_{\infty} t_{c h}\right)^{1 / 2}$, be of the order of $u_{N S}$ or larger. If $t_{d}$ is shorter than $t_{c h}$ the chemical reaction has no time to occur in the aforesaid regions, and, as mentioned in the previous subsection, a steady triple flame cannot exist in Goldstein's shear layer immediately behind either, because the velocity of the flow here is larger than the velocity of propagation of the flame. In these conditions the flame lifts off the injector rim or is blown out completely. On the other hand, the triple flame always finds an equilibrium position in the Navier-Stokes region if $t_{d}$ is larger than $t_{c h}$, because, owing to the heat losses toward the solid, the excess of enthalpy ( $H$; cf. (2.3)) is negative in this region and the temperature of the front gets smaller than the adiabatic flame temperature, increasing drastically the chemical time. Then the flame front provides a continuous ignition source for the diffusion flame downstream.

The mechanism of flame stabilization seems to be somewhat different when both fluids move and the thickness of the separating wall is much larger than $\delta_{N S}$, and the Navier-Stokes regions then play a less prominent role. This can be seen by analysing the flow about the edge. At a distance $r \gg \delta_{N S}$ from the edge the flux carried by Goldstein's shear layer is of order $\rho_{\infty} u_{N S} \delta_{N S}\left(r / \delta_{N S}\right)^{2 / 3}$ and the entrainment of this layer leads to an effectively inviscid flow below, with velocity $O\left[u_{N S}\left(r / \delta_{N S}\right)^{-1 / 3}\right]$, and to a boundary layer of thickness $\delta_{N S}\left(r / \delta_{N S}\right)^{2 / 3}$ on the vertical side of the wall. With a flame present, the fluid entering this boundary layer comes from regions of high temperature downstream and loses heat toward the wall, which results in a deficiency of enthalpy. Further, most of this fluid leaves the boundary layer before reaching the NavierStokes region (the flux in the boundary layer is of order $\rho_{\infty} u_{N S} \delta_{N S}\left(r / \delta_{N s}\right)^{1 / 3}$, decreasing with $r$ ) and is ingested by the shear layer after crossing the inviscid region in between. On a wall of thickness $h\left(\gg \delta_{N s}\right)$, the maximum thickness attained by this boundary layer is of order $\delta_{N S}\left(h / \delta_{N S}\right)^{2 / 3}$ and the corresponding flux, $O\left[\rho_{\infty} u_{N S} \delta_{N S}\left(h / \delta_{N S}\right)^{1 / 3}\right]$, is ingested by the shear layer at a distance of order $\delta_{N S}\left(h / \delta_{N S}\right)^{1 / 2}$ from the solid. This defines the length of the attachment region, over which the enthalpy of the flow in the shear layer, as well as its velocity, is an increasing function of the distance from 
the wall. Notice, however, that for a triple flame to exist here its propagation velocity has to match that of the flow, which is faster than in the Navier-Stokes region.

The solutions in the different regions mentioned above can be easily obtained as follows. First, in the inviscid recirculation region $x_{1}>0,|y| \leqslant 1$, with the streamwise distance now scaled with $h$, the stream function $\psi_{1}$ (scaled with $\left[\lambda_{F 0} h^{2} \mu_{\infty}^{2} / \rho_{\infty}\right]^{1 / 3}$ ) obeys $\nabla^{2} \psi_{1}=-\rho_{b} \mathscr{A} \psi_{1}$ with the boundary conditions $\psi_{1}=0$ at $x_{1}=0 ; \partial \psi_{1} / \partial x_{1}=$ $\pm \frac{2}{3}\left(f_{u, l} / x_{1}^{1 / 3}\right)$ at $y= \pm 1$; and $\psi_{1} \rightarrow x_{1}^{2 / 3} F(y)$ for $x_{1} \rightarrow \infty$, where $\rho_{b}, \mathscr{A}, f_{u}, f_{l}$, and $F(y)$ are defined in $\S 3$. The solution of this problem determines the slip velocity $\left(1 / \rho_{b}\right)\left(\partial \psi_{1} / \partial x_{1}\right)_{x_{1}=0}$ driving the flow in the boundary layer on the rim of the injector. Second, knowing this velocity and the temperature $T_{b}$ of the fluid outside the boundary layer, and assuming that the temperature of the solid is $T=1$, the velocity and temperature in the boundary layer can be immediately computed for $y_{0}<y<1$ and for $-1<y<y_{0}$. This solution determines the temperature distributions, $T_{u, l}\left(\psi_{2}\right)$ say, for $y \rightarrow \pm 1$, where $\psi_{2}$ is the stream function in the boundary layer (scaled with $\left.\rho_{\infty} u_{N S} \delta_{N s}\left(h / \delta_{N s}\right)^{1 / 3}\right)$. Third, the flow in the upper shear layer upstream of the flame front (the lower shear layer is similar) obeys the boundary layer equations (2.6)-(2.9) without pressure gradient, plus an equation similar to (2.8) for the enthalpy excess $H$ defined in (2.3), subject to the boundary conditions

$$
\frac{\partial \psi_{2}}{\partial y_{2}}=\alpha\left(y_{2}+0\right), \quad Z=0, \quad H=0 \quad \text { for } \quad y_{2} \rightarrow \infty
$$

and

$$
\frac{\partial \psi_{2}}{\partial y_{2}}=0, \quad Z=Z_{b}, \quad H=T_{u}\left(\psi_{2}\right)-T_{b} \quad \text { for } \quad y_{2} \rightarrow-\infty,
$$

where $y_{2}=(y-h) /\left[\delta_{N S}\left(h / \delta_{N S}\right)^{1 / 6}\right]$ is of order unity in the shear layer for $x_{2}=$ $x /\left[\delta_{N S}\left(h / \delta_{N S}\right)^{1 / 2}\right]=O(1)$. The solution of this problem determines the distributions of velocity, $Z$, and $H$ ahead of the flame, where the mixture fractions of the reactants and the temperature are $Y_{o}=\left(Z_{b}-Z\right) / Z_{b}, Y_{F}=\left(Z / Z_{b}\right)\left(Z_{b}-Z_{s}\right) /\left(1-Z_{s}\right)$, and $T=1+H-\gamma\left(Y_{F}+Y_{o}-1\right)$. Finally, with these data, a local analysis along the lines of Liñán (1994) would determine if a triple flame matching the velocity of the fluid can exist at some section of the shear layer.

By way of example, this program was carried out for $\alpha=1$ and $\gamma \rightarrow 0$, in which limit $f_{u}=f_{l} \approx-1.258$, the recirculating flow and the boundary layers are symmetric, and the velocity in the shear layers is self-similar, not being affected by the presence of the flame. If the flame front were to sit at a certain position in one of the shear layers, the concentration of the rich reactant on the flame and the flame temperature, obtained from (2.3) with the condition that the concentration of the lean reactant be zero, would be given by the first relations in (2.5) and

$$
T_{f}=\left\{\begin{array}{lll}
1+\gamma Z / Z_{s}+H & \text { for } & 0 \leqslant Z \leqslant Z_{s} \\
1+\gamma \frac{1-Z}{1-Z_{s}}+H & \text { for } & Z_{s} \leqslant Z \leqslant 1,
\end{array}\right.
$$

respectively.

The scaled temperature rise $\left(T_{f}-1\right) /\left(T_{e}-1\right)$ as a function of $\left(x_{2}, y_{2}\right)$ is shown in figure 9 for $S=4$. In this particular case the maximum temperature in each section is attained where $Z=Z_{s}$. The velocity of the nose of the flame front, equal to the burning rate of the corresponding planar premixed flame, is a rapidly increasing function of $T_{f}$ and depends more weakly on the concentration of the rich reactant on the flame 


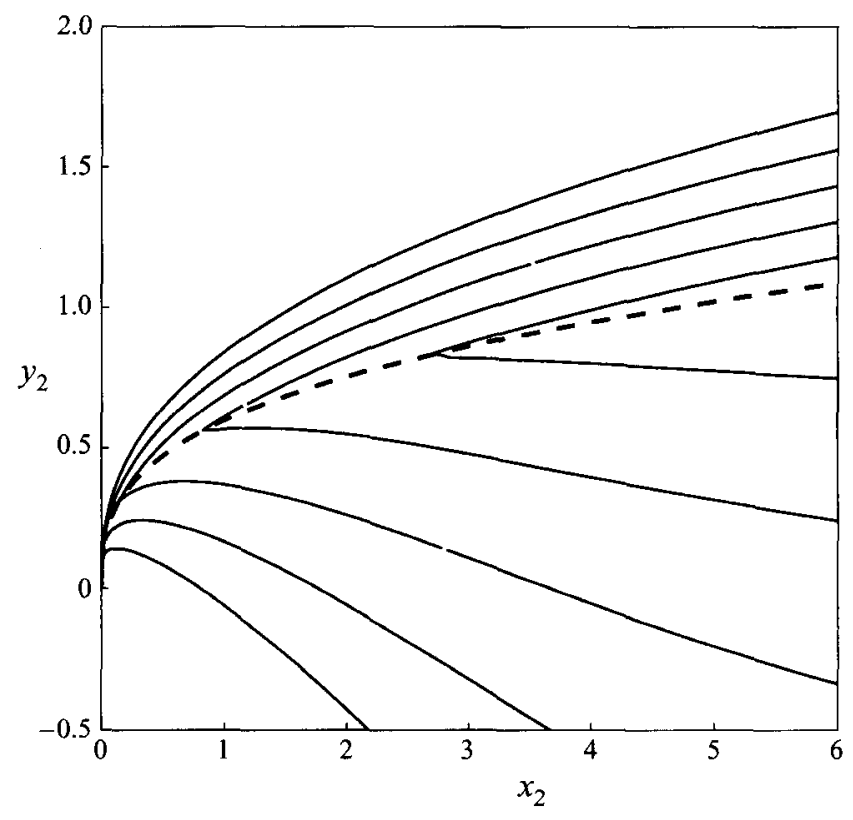

FIGURE 9. Increment of temperature at the flame in the region of enthalpy defect for $\operatorname{Pr}=\alpha=1$, $S=4$, and $\gamma \rightarrow 0$. Contours plotted are $\left(T_{f}-1\right) /\left(T_{e}-1\right)=0.7,0.75,0.8,0.85$, and 0.9 , increasing from left to right. Dashed: contour $Z=Z_{s}$.

(see, e.g., Williams 1985), so the point in the shear layer where this velocity matches the velocity of the fluid can be easily determined for any given burning rate law.

Two other factors that might facilitate the attachment of the flame can be mentioned. Firstly, the heat transferred by the recirculating fluid to the solid may be recovered by the oncoming streams of reactants, at a large distance from the end of the wall if its conductivity is high, in such a way that the enthalpy is merely redistributed and the enthalpy defect of the flow entering the shear layer from the recirculation region can be balanced by the enthalpy excess of the flow entering from the reactant stream. Secondly, the velocity of the flow in the region of the shear layer containing the flame may be appreciable lowered by the effect of the gas expansion for sufficiently large values of $\gamma$ (cf. $\S 4)$.

\section{REFERENCES}

Gaydon, A. G. \& Wolfhard, H. G. 1953 Flames-Their Structure, Radiation, and Temperature, p. 39. Chapman \& Hall.

GoLDSTEIN, S. 1930 Concerning some solutions of the boundary-layer equations in hydrodynamics. Proc. Camb. Phil. Soc. 26, 1-30.

HakkInen, R. J. \& RotT, N. 1965 Similarity solutions for merging shear flows II. AIAA J. 3, $1553-1554$.

KaWAMura, T. \& Asato, K. 1975 The structure of the stabilizing region of a laminar fuel jet flame. Proc. 2nd European Symp. on Combustion, pp. 611-616. The Combustion Institute, Pittsburgh.

Kawamura, T., Asato, K. \& MazaKi, T. 1980 Structure analysis of the stabilizing region of plane, laminar fuel-jet flames. Combust. Sci. Tech. 22, 211-216.

Lewis, B. \& ElBE, G. vON 1961 Combustion, Flames and Explosions of Gases, 3rd. edn., pp. 233-236. Academic Press.

LIÑÁN, A. 1974 The asymptotic structure of laminar diffusion flames for large activation energies. Astro. Acta 1, 1007-1031. 
LiÑÁN, A. 1994 Ignition and flame spread in laminar mixing layers. In Combustion in High Speed Flows (ed. J. Buckmaster et al.), pp. 461-476. Kluwer.

Messiter, A. F. 1970 Boundary layer flow near the trailing edge of a flat plate. SIAM J. Appl. Maths 18, 241-257.

Robson, K. \& WiLson, M. J. G. 1969 The stability of laminar diffusion flames of methane. Combust. Flame 13, 626-634.

SмIт, F. T. 1982 On the high Reynolds number theory of laminar flows. IMA J. Appl. Maths 28, 207-281.

STEWARTSON, K. 1969 On the flow near the trailing edge of a flat plate II. Mathematika 16, 106-121.

Takahashi, F., Mizomoto, M., IKaI, S. \& Futaki, N. 1984 Lifting mechanism of free jet diffusion flames. 20th Symp. on Combustion, pp. 295-302. The Combustion Institute.

TaKahashi, F. \& Schmoll, W. J. 1990 Lifting criteria of jet diffusion flames. 23rd Symp. on Combustion, pp. 677-683. The Combustion Institute.

Vatsa, V. N., Werle, M. J. \& Verdon, J. M. 1981 Analysis of laminar and turbulent blunt trailing-edge flows. Report R81-914986-5. United Tech. Res. Center, East Hartford, Conn.

Vatsa, V. N., Werle, M. J. \& Verdon, J. M. 1982 Viscid-inviscid interaction at laminar and turbulent symmetric trailing edges. AIAA Paper 82-0165.

VRanos, A., Taback, E. D. \& Shipman, C. W. 1968 An experimental study of the stability of hydrogen-air diffusion flames. Combust. Flame 12, 253-260.

Williams, F. A. 1985 Combustion Theory, 2nd edn., chapter 3. Benjamin/Cummings.

Yoon, Y., Donbar, J. M. \& Driscoll, J. F. 1994 Blowout stability limits of a hydrogen jet flame in a supersonic, heated, coflowing air stream. Combust. Sci. Tech. 97, 137-156. 